\title{
The Influence Analysis of Number of Functional Logistics Service Providers on Quality Supervision Game in LSSC with Compensation Strategy
}

\author{
Weihua Liu, Yijia Wang, Zhicheng Liang, and Xiaoyan Liu \\ School of Management, Tianjin University, Tianjin 300072, China \\ Correspondence should be addressed to Weihua Liu; lwhliu888@163.com
}

Received 14 October 2013; Revised 15 February 2014; Accepted 15 February 2014; Published 31 March 2014

Academic Editor: Rafael Jacinto Villanueva

Copyright (c) 2014 Weihua Liu et al. This is an open access article distributed under the Creative Commons Attribution License, which permits unrestricted use, distribution, and reproduction in any medium, provided the original work is properly cited.

\begin{abstract}
There is a close link between the number of suppliers and the quality of products including service. However, there is a research gap in this field. Particularly, the problem whether this link would be affected by different supply chain profit distribution policies is lack of in-depth research. In this paper, a basic game model for quality supervision game without FLSPs' competition (Model I) in a logistics service supply chain (LSSC) is established first. Model I adopts a mixed payment contract and is composed of a logistics service integrator (LSI) and functional logistics service provider (FLSP). The mixed-strategy Nash equilibrium of Model I is presented. Model II considering competition among FLSPs is then built based on Model I, and the new mixedstrategy Nash equilibrium is provided. Results show that under competition the ordinary mixed payment contract cannot make quality supervision game parameters all optimized. Therefore, Model III under a compensation mechanism is established based on Model II, and the range of the compensation value is calculated. Furthermore, we propose three kinds of concrete compensation mechanisms, which are fixed, linear, and nonlinear compensation mechanism. And the optimal compensation mechanism for a LSI with different numbers of FLSPs is provided.
\end{abstract}

\section{Introduction}

In recent years, service outsourcing such as IT service outsourcing, financial service outsourcing, and logistics service outsourcing is becoming increasingly popular. Many service integrators have established long-term partnership with service providers to provide customers with integrated services. In the process, service supply chains have been formed. Take logistics services outsourcing as an example; a logistics service integrator (LSI) integrates multiple functional logistics service providers' (FLSP) service capabilities, providing customers with integrated logistics services, and the LSI and FLSP form the logistics service supply chain [1]. For example, P\&G Logistics Group Co., Ltd., in Guangzhou, as the largest LSI in China, successfully undertakes the logistics business of its customers (Procter \& Gamble, Philips, etc.) by integrating more than 500 warehousing suppliers, 1,200 road transport suppliers, and 500 manpower loading and unloading operation teams.
A supply chain is a typical system that requires coordination, and LSSC is not an exception. LSSC is a service supply chain whose core is capacity cooperation [1]. The LSI strengthening the quality supervision is the key factor to achieving successful cooperation within a service supply chain [2]. As the LSI usually has to integrate the service capabilities of multiple FLSPs when providing logistics services for customers, not only would the number of FLSPs affect whether the demand for services can be satisfied successfully but also the competition among them would directly affect the LSI's quality supervision results. The existing supply chain quality management research results show that there is a close link between the number of suppliers and the quality of products (services) [3]. On the other hand, there is a research gap in the relationship between the number of suppliers and the quality supervising strength of manufacturers (or service integrators). In particular, an in-depth research has not yet been conducted on the question whether different supply 
chain profit distribution policies would have an impact on these two factors.

The necessity of this study in the industrial practice has also been confirmed. From the industrial practice, many LSIs in China, such as Baogong logistics company, Tianjin Baoyun Logistics company, and Beijing Leader logistics company, obtain a high level of service quality via reinforcing the competition among multiple FLSPs in the process of completing logistics service orders in the goal of achieving optimal service performance. The competition among multiple FLSPs indeed helps to reduce the LSI's supervision difficulty level of logistics service quality, but it does not significantly improve the FLSPs' logistics service quality and the cooperation initiatives among them. P\&G Logistics Group Co., Ltd., for example, had to replace at least $10 \%$ of the FLSPs by the end of each year in order to improve the service quality the following year [4]. This leads us to an interesting question: why the competition among multiple FLSPs does not effectively improve the logistics service quality? Can we introduce a kind of profit compensation mechanism to reduce the LSI's supervision difficulty level as well as improve FLSP' service quality? Assuming this kind of profit compensation mechanism exists, what is the relationship between the kind of mechanism and the number of FLSPs? These issues, which have not been studied and discussed in the industrial practice, are to be discussed in depth in this paper.

Considering the difficulty of measuring service, a model (Model I) for quality supervision game in LSSC without FLSPs' competition was initially established in this study based on the model developed by Liu and Xie [5]. Model I assumes that the supply chain adopts a mixed payment contract and the optimal supervision probability of the LSI and the optimal compliance probability of FLSP $i$ are obtained. Based on Model I and taking the impact of competition among FLSPs on the effect of quality supervision game into consideration [3], we introduced the competition factor to establish Model II (the quality supervision game model for LSSC with FLSPs' competition). By solving and analyzing Model II, we found that, under competition, the ordinary mixed payment contract cannot make quality supervision game parameters all optimized. Thus, we introduced compensation mechanism to optimize the effect of supply chain quality supervision and established Model III: the quality supervision game model with FLSP' competition under a compensation mechanism. We have obtained some important findings from the study of Model III. For example, when the number of FLSPs is small, the LSI should choose nonlinear compensation mechanism; when the number of FLSPs is large, the LSI should choose linear compensation mechanism; when the number of FLSPs is in other intervals, no optimal compensation mechanism exists. In the third scenario, the LSI has to make a trade-off between the LSI's optimal supervision probability and FLSPs' optimal compliance probability when choosing a compensation mechanism. Figure 1 is the research process in this paper.

This paper is organized as follows. Section 2 provides the literature review of this paper. The quality supervision game model for LSSC without FLSPs' competition (Model I) is presented in Section 3 and the Nash equilibrium is obtained.
In this section, the quality supervision game model for LSSC with FLSPs' competition (Model II) is presented by introducing competition among FLSPs. Via introducing compensation mechanism, the quality supervision game model with FLSPs' competition under a compensation mechanism (Model III) is presented in Section 4. MATLAB 8.0 software is utilized for numerical analysis in Section 5 to verify the main conclusions. The conclusions and management implications of this paper are presented in Section 6.

\section{Literature Review}

The existing research on the impact of the number of competitors on the quality supervision game for a supply chain mainly focuses on the manufacturing supply chain. However, research on the service supply chain, especially LSSC, is relatively inadequate. This section (Literature Review) mainly includes three aspects: (1) review of the literature on supply chain quality supervision game, (2) summary of research on competition among providers for manufacturing supply chain, and (3) review of the literature on compensation contract.

2.1. Supply Chain Quality Supervision Game. Quality issues in the supply chain, mostly those with regard to the significance and importance of quality in the manufacturing supply chain $[6,7]$ and the contract design of quality control, have been discussed extensively in relevant literature. The International Journal of Production Economics published a special issue for supply chain quality management including eight papers that focused on theoretical models and empirical studies [4]. The risk involved in quality control game has also been discussed in recent years. For example, Baiman et al. discussed quality control game based on the moral hazard in supply chain cooperation [8]. Lim established a quality control model with asymmetric information [9]. Kaya and Özer discussed the quality risk of outsourcing [10]. Chao et al. discussed two contractual agreements by which product recall costs can be shared between the manufacturer and suppliers to induce quality improvement for cases where information on the quality of suppliers' products is not accessible to the manufacturer [11].

The increase in the number of studies on service supply chain has caused quality control game in LSSC to be continuously studied. Hertz and Macquet established a Nash equilibrium model for the third-party logistics service providers and subcontractors and analyzed the impact on quality control as contract parameters change [12]. Jayaram and Tan believed that supervising the service quality of the third-party logistics (TPL) is important [13]. Considering that the quality of logistics services is difficult to measure, Liu et al. developed a multiperiod quality control game model in twoechelon LSSC and a model in three-echelon LSSC [2]. Liu and Xie studied the quality decision problems of LSIs and FLSPs when FLSPs provide service quality guarantee [5]. However, these studies did not consider the influence of the number of FLSPs on quality decisions. 


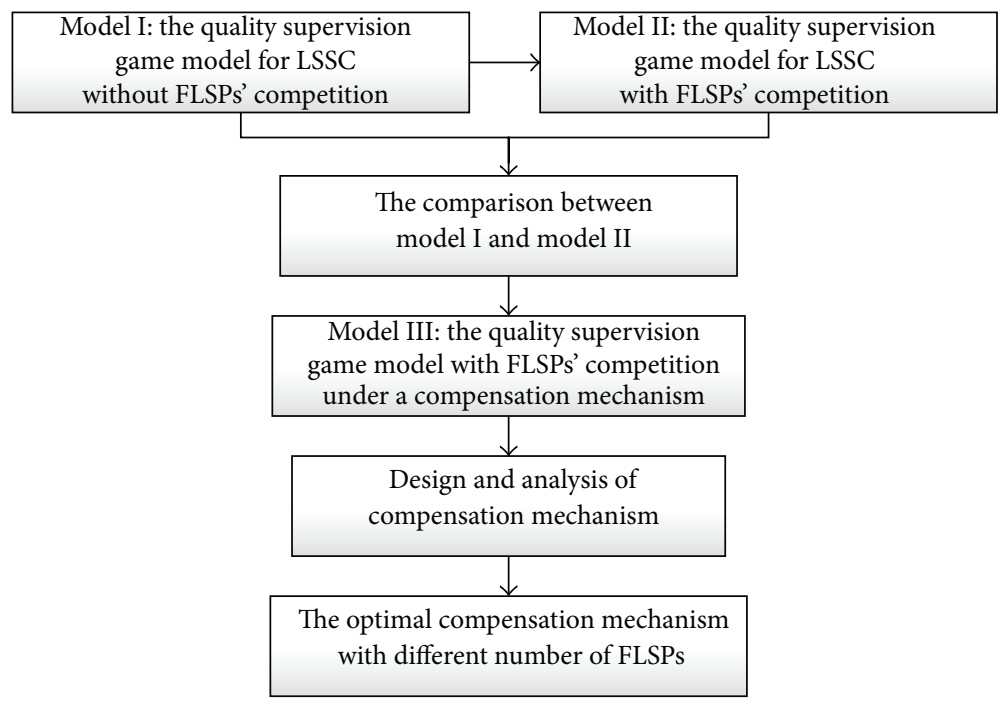

FIGURE 1: The research process of this paper.

2.2. The Impact of FLSP' Competition on the Supply Chain Performance. The study on the impact of providers' competition on the supply chain mainly focuses on the decision of the providers' number. Some literature shows that the integrator gains advantage by owning multiple providers whether requirements are determined or stochastic [14-16]. Many scholars discussed how to determine the optimal number of suppliers, and the common modeling methods include decision trees $[17,18]$ and goal programming method $[19,20]$. In recent years, many literatures have discussed the supplier quantity decision problem in a competitive environment [21-23], considering that the number of businesses of each supplier is related to that of others.

As for service integrators, at present, researches mainly focus on selection and evaluation of the service outsourcing suppliers, and the selection of multiservice outsourcing suppliers is considered as well as the multisourcing suppliers selection $[24,25]$. On the basis of the basic quality control game model in a two-echelon logistics service supply chain, Liu et al. considered the impact of the competition among FLSPs on the quality control and found that the quality control can be strengthened by reinforcing monitoring and control in the process of logistics cooperation and establishing a competition mechanism among FLSPs and so forth [2]. But there is little literature regarding how to design a contract to improve the efficiency of the supply chain with a certain number of providers.

2.3. Compensation Contract. Compensation contract is widely used in supply chain coordination. It can be used to overcome information asymmetry between buyers and sellers [26], to motivate retailers to increase the order quantity [27], to increase sales effort [28, 29], and to improve the performance of decentralized supply chains [30-32]. In addition, Starbird studied the award, punishment, and supervision strategies that the buyer applies to suppliers' quality, and he found that reward and punishment can be substituted for each other to motivate providers [33]. Zhang et al. compared buyback contract, target compensation contracts, and incremental buyback contract when the retailer is risk-averse [34]. In the model presented by Taylor, there were two forms of compensation, linear (pay a certain amount of compensation per unit) and nonlinear (pay a certain amount of compensation per unit when a certain sales target is reached) [29]. The study showed that compensation would improve the retailer's sales effort. However, these studies about compensation have rarely been applied to service supply chain, and few scholars have applied compensation contract to improve the supply chain performance when considering competition among providers.

Based on the literature review above, we have found that the existing research has two deficiencies as follows.

(1) Research on supply chain quality supervision game mainly focuses on product supply chain, and research on quality supervision-oriented service supply chain is inadequate, especially the research on the impact of multiple FLSPs' competition on the effect of quality supervision in service supply chain.

(2) The existing research on the quality supervision game in LSSC explored the impact of quality behaviors (such as quality commitment behavior) of a LSI and a FLSP on the performance of the supply chain but did not consider the impact of multiple FLSP' competition on the performance of the supply chain $[2,5]$.

This study aims to address the two problems mentioned above. We have established a quality supervision game model for LSSC with FLSPs' competition. Then by introducing compensation mechanism, the quality supervision game model with FLSPs' compensation under a compensation mechanism is presented, and the relationship between the number of FLSPs and the type of compensation mechanisms 
is discussed. The results of this research can serve as a scientific reference for quality decision making of LSIs.

\section{The Quality Supervision Game Models for LSSC without Compensation}

Some of the important assumptions and variables of Models I and II are provided in Section 3.1. The quality supervision game model for LSSC without FLSPs' competition (model I) is established in Section 3.2. The section also presents the optimal supervision probability of the LSI and the optimal compliance probability of the FLSP. Based on Model I, the quality supervision game model for the LSSC with FLSPs' competition (Model II) is studied in Section 3.3. And the analytical results of Model II are compared with that of Model II in this section.

3.1. Assumptions and Parameters. We assume that a LSSC is composed of a LSI and a FLSP, and the LSI is in dominant position. The logistics capacity required by the LSI is completely provided by the FLSP, and the FLSP has the ability to complete the LSI's outsourced tasks. The FLSP's effort level determines its service quality, and its service quality directly determines the total revenue of the LSSC. Some of the assumptions of the model are described below.

Assumption 1. The FLSP provides logistics services for the LSI. The total revenue $\pi(p)$ of the LSSC is a function of the FLSP's service quality, and the FLSP's service quality is a function of the FLSP's effort level $p$. We assume that $\pi(p)=$ $g(p)+\varepsilon$ and $\varepsilon \sim N\left(0, \sigma_{\varepsilon}^{2}\right)$ in which $g(p)$ is a function of $p$ and $\varepsilon$ is the impact of the environment on total revenue.

Assumption 2. The FLSP in cooperation with the LSI has two choices: comply with or break the contract. The service cost $c\left(p_{i}\right)$ of the FLSP is a function of its effort level. When the FLSP complies with the contract, the effort level is $p_{1}$, the total revenue of the LSSC is $\pi\left(p_{1}\right)=g\left(p_{1}\right)+\varepsilon$, and the service cost of the FLSP is $c\left(p_{1}\right)$. When the FLSP opts to break the contract, the effort level is $p_{2}$, the total revenue of the LSSC is $\pi\left(p_{2}\right)=g\left(p_{2}\right)+\varepsilon$, and the service cost of the FLSP is $c\left(p_{2}\right)$. The FLSP can acquire a certain amount of self-interest $\varphi$ from breaking the contract.

Assumption 3. The LSI also has two choices: to supervise the service quality of the FLSP or not. When the LSI supervises quality, the supervision cost $f(q)$ is not only associated with its supervision effort but is also affected by the external environment. We let $f(q)=h(q)+\eta$ and $\eta \sim N\left(0, \sigma_{\eta}^{2}\right)$, where $h(q)$ is a function of $q, q$ is the supervision effort level of the LSI, and $\eta$ is the impact of the environment on the LSI's supervision cost. Given that service is difficult to measure [35], breach of the contract by the FLSP may not be discovered by the LSI. The probability of the FLSP breaking the contract and being discovered is assumed to be $\rho$, and the penalty of this situation is $\theta$.
Assumption 4. We assume that the LSI provides a mixed payment contract to the FLSP. The revenue of the FLSP in the contract can be divided into two parts. One is a fixed payment, and the other is a specific percentage of the LSSC's total net income, which is equal to $\omega+r[\pi(p)-\omega]$. Here, $p$ represents the FLSP's effort level, $\omega$ is the fixed payment, and $r$ is the coefficient of the revenue that the FLSP obtains from cooperation. Accordingly, the income of the LSI is (1 $r)[\pi(p)-\omega]$.

Assumption 5. $\varepsilon$ and $\eta$ are independent of each other; that is, $\operatorname{Cov}(\varepsilon, \eta)=0$.

The notations for the model are summarized in the Appendix.

3.2. Model I: The Quality Supervision Game Model for LSSC without FLSPs' Competition. When the FLSP opts to comply with the contract, the effort level of the FLSP is $p_{1}$, the service cost is $c\left(p_{1}\right)$, the total revenue of the LSSC is $\pi\left(p_{1}\right)$, and the expected revenue of the FLSP is $(1-r) \omega+r \pi\left(p_{1}\right)-c\left(p_{1}\right)$. When the LSI supervises service quality, the supervision cost is $f(q)$ and expected revenue is $(1-r)\left[\pi\left(p_{1}\right)-\omega\right]-f(q)$; otherwise, the expected revenue is $(1-r)\left[\pi\left(p_{1}\right)-\omega\right]$.

When the FLSP opts to break the contract, the effort exerted by the FLSP is $p_{2}$, the service cost is $c\left(p_{2}\right)$, the total revenue of the LSSC is $\pi\left(p_{2}\right)$, and the FLSP obtains personal interests $\varphi$. When the LSI supervises service quality, the supervision cost is $f(q)$, the probability of the FLSP's breach of the contract being discovered is $\rho$, and the FLSP's penalty from the LSI is $\theta$. The expected revenue of the FLSP is $(1-r) \omega+r \pi\left(p_{2}\right)-c\left(p_{2}\right)+\varphi-\rho \theta$, and the expected revenue of the LSI is $(1-r)\left[\pi\left(p_{2}\right)-\omega\right]-f(q)+\rho \theta$. When the LSI does not supervise service quality, the expected revenue of the FLSP is $(1-r) \omega+r \pi\left(p_{2}\right)-c\left(p_{2}\right)+\varphi$, and the expected revenue of the LSI is $(1-r)\left[\pi\left(p_{2}\right)-\omega\right]$. The expected payoff matrix is shown in Table 1 , in which $(1-r) \omega+r \pi\left(p_{2}\right)-c\left(p_{2}\right)+\varphi>$ $(1-r) \omega+r \pi\left(p_{1}\right)-c\left(p_{1}\right)$.

From Table 1, we can see that a pure strategy Nash equilibrium does not exist. Therefore, we proceed to find a mixed strategy Nash equilibrium. We assume that the supervision probability of the LSI is $x$, nonsupervision probability is $(1-x)$, compliance probability of the FLSP is $y$, and noncompliance probability is $(1-y)$. The expected revenue of the LSI is

$$
\begin{aligned}
& \Pi_{I}^{0}(x, y) \\
& =x\left\{y\left[(1-r)\left(\pi\left(p_{1}\right)-\omega\right)-f(q)\right]\right. \\
& \left.+(1-y)\left[(1-r)\left(\pi\left(p_{2}\right)-\omega\right)-f(q)+\rho \theta\right]\right\} \\
& +(1-x) \\
& \times\left\{y\left[(1-r)\left(\pi\left(p_{1}\right)-\omega\right)\right]+(1-y)\left[(1-r)\left(\pi\left(p_{2}\right)-\omega\right)\right]\right\} \\
& =[\rho \theta-f(q)] x+(1-r)\left[\pi\left(p_{1}\right)-\pi\left(p_{2}\right)\right] y \\
& -\rho \theta x y+(1-r) \pi\left(p_{2}\right)-(1-r) \omega .
\end{aligned}
$$


The maximum expected revenue of the LSI meets the condition that $\partial \Pi_{I}^{0}(x, y) / \partial x=0$; that is,

$$
y_{0}^{*}=\frac{\rho \theta-f(q)}{\rho \theta}=\frac{A}{\rho \theta} .
$$

Equation (2) shows that $y_{0}^{*}$ is positively correlated to $\rho$ and $\theta$ and negatively correlated to $f(q)$.

The expected revenue of the FLSP is

$$
\begin{aligned}
& \Pi_{F}^{0}(x, y) \\
& =x\left\{y\left[(1-r) \omega+r \pi\left(p_{1}\right)-c\left(p_{1}\right)\right]\right. \\
& \left.\quad+(1-y)\left[(1-r) \omega+r \pi\left(p_{2}\right)-c\left(p_{2}\right)+\varphi-\rho \theta\right]\right\} \\
& \quad+(1-x) \\
& \quad \times\left\{y\left[(1-r) \omega+r \pi\left(p_{1}\right)-c\left(p_{1}\right)\right]+(1-y)\right. \\
& \left.\quad \times\left[(1-r) \omega+r \pi\left(p_{2}\right)-c\left(p_{2}\right)+\varphi\right]\right\} \\
& =-\rho \theta x+\left\{r\left[\pi\left(p_{1}\right)-\pi\left(p_{2}\right)\right]-\left[c\left(p_{1}\right)-c\left(p_{2}\right)\right]-\varphi\right\} y \\
& +\rho \theta x y \\
& +(1-r) \omega+r \pi\left(p_{2}\right)-c\left(p_{2}\right)+\varphi .
\end{aligned}
$$

The maximum expected revenue of the FLSP meets the condition that $\partial \Pi_{F}^{0}(x, y) / \partial y=0$; that is,

$$
x_{0}^{*}=\frac{\varphi-r\left[\pi\left(p_{1}\right)-\pi\left(p_{2}\right)\right]+\left[c\left(p_{1}\right)-c\left(p_{2}\right)\right]}{\rho \theta}=\frac{-T}{\rho \theta} .
$$

Equation (4) shows that $x_{0}^{*}$ is negatively correlated to $\rho$, $\theta, r$, and $\left[\pi\left(p_{1}\right)-\pi\left(p_{2}\right)\right]$ and positively correlated to $\varphi$ and $\left[c\left(p_{1}\right)-c\left(p_{2}\right)\right]$.

3.3. Model II: The Quality Supervision Game Model for LSSC with FLSPs' Competition. Now the model will be extended to the case of multiple FLSPs. It is assumed that the LSI has multiple upstream FLSPs, and there is mutual influence among FLSPs. We assume that the LSI takes the following punishment strategy: if FLSP $i$ cannot achieve the required service quality according to agreement, then the LSI will choose other FLSPs, reduce the cooperation level with FLSP $i$, and even ultimately abandon the cooperation with FLSP $i$. Therefore, the presence of competitors makes any FLSP's default cost increase and expected revenue decrease accordingly. This loss of FLSP $i$ is $F_{i}(N)=\gamma N b R$, where $b$ denotes the competitive coefficient, that is, the degree of mutual influence among FLSPs, and $0<b<1$. A large $b$ means that the business competition among FLSPs is fierce; in particular, there is not any mutual effect among FLSPs when $b=0$. $R$ indicates the service quality level of FLSP $i$, which can be informed from the FLSP's past experience of cooperation or credibility. It is assumed in this paper that all the FLSPs are homogeneous; that is, their service qualities are at the same level. The expected revenue matrix of the LSI and FLSP $i$ considering FLSP's competition is shown in Table 2.
The expected revenue function of the LSI in this case is

$$
\begin{aligned}
\Pi_{I}^{1}(x, y)= & \Pi_{I}^{0}(x, y) \\
= & {[\rho \theta-f(q)] x+(1-r)\left[\pi\left(p_{1}\right)-\pi\left(p_{2}\right)\right] y } \\
& -\rho \theta x y+(1-r) \pi\left(p_{2}\right)-(1-r) \omega .
\end{aligned}
$$

We let $\partial \Pi_{I}^{1}(x, y) / \partial x=0$ to obtain

$$
y_{1}^{*}=\frac{A}{\rho \theta} .
$$

The expected revenue function of FLSP $i$ is

$$
\begin{aligned}
& \Pi_{F_{i}}^{1}(x, y) \\
& =\Pi_{I}^{0}(x, y)-(1-y) F_{i}(N) \\
& =-\rho \theta x+\left\{r\left[\pi\left(p_{1}\right)-\pi\left(p_{2}\right)\right]-\left[c\left(p_{1}\right)-c\left(p_{2}\right)\right]\right. \\
& \quad-\varphi+\gamma N b R\} y \\
& \quad+\rho \theta x y+(1-r) \omega+r \pi\left(p_{2}\right)-c\left(p_{2}\right)+\varphi-\gamma N b R .
\end{aligned}
$$

We let $\partial \Pi_{F_{i}}^{1}(x, y) / \partial y=0$ to obtain

$$
x_{1}^{*}=\frac{-T-\gamma N b R}{\rho \theta} .
$$

Comparing (2), (4), (6), and (8), we find that when taking into consideration the competition among FLSPs, the mixed payment contract reduces the LSI's optimal supervision probability, but it does not increase FLSP i's optimal compliance probability.

\section{Model III: The Quality Supervision Game Model with FLSPs' Competition under a Compensation Mechanism}

The analysis in Section 3.3 shows that, under the abovementioned mixed payment contract, the introduction of FLSPs' competition makes the LSI's optimal supervision probability decrease, but FLSP is optimal compliance probability remains unchanged. In Section 4.1, we derive a compensation mechanism which optimizes the LSI's optimal supervision probability, FLSP $i$ 's optimal compliance probability, and the expected revenue of the LSI and FLSP $i$. In Section 4.2, we design three specific compensation mechanisms, that is, fixed compensation mechanism, linear compensation mechanism, and nonlinear compensation mechanism, and calculate their respective range of application. Then a comparison is made among the three compensation mechanisms in Section 4.3, and the results can serve as a scientific reference for compensation decision making in the LSI.

4.1. Modeling and Solving. It is assumed that $\alpha(\alpha>0)$ is the compensation that the LSI pays to FLSP $i$ when FLSP $i$ complies with the contract (including the situation that FLSP $i$ 's breach of the contract is not discovered and that FLSP $i$ 
TABLE 1: Expected revenue matrix of the LSI and the FLSP.

\begin{tabular}{|c|c|c|}
\hline \multirow{2}{*}{ LSI } & \multicolumn{2}{|c|}{ FLSP } \\
\hline & Complies with the contract & Breaks the contract \\
\hline Supervises & $\begin{array}{l}(1-r) \omega+r \pi\left(p_{1}\right)-c\left(p_{1}\right) \\
(1-r)\left[\pi\left(p_{1}\right)-\omega\right]-f(q)\end{array}$ & $\begin{array}{l}(1-r) \omega+r \pi\left(p_{2}\right)-c\left(p_{2}\right)+\varphi-\rho \theta \\
(1-r)\left[\pi\left(p_{2}\right)-\omega\right]-f(q)+\rho \theta\end{array}$ \\
\hline Does not supervise & $\begin{array}{l}(1-r) \omega+r \pi\left(p_{1}\right)-c\left(p_{1}\right) \\
(1-r)\left[\pi\left(p_{1}\right)-\omega\right]\end{array}$ & $\begin{array}{l}(1-r) \omega+r \pi\left(p_{2}\right)-c\left(p_{2}\right)+\varphi \\
(1-r)\left[\pi\left(p_{2}\right)-\omega\right]\end{array}$ \\
\hline
\end{tabular}

TABLE 2: Expected revenue matrix of the LSI and FLSP $i$ considering FLSP's competition.

LSI

\begin{tabular}{ll} 
& Complies with the contract \\
\hline \multirow{2}{*}{ Supervises } & $(1-r) \omega+r \pi\left(p_{1}\right)-c\left(p_{1}\right)$, \\
& $(1-r)\left[\pi\left(p_{1}\right)-\omega\right]-f(q)$ \\
& $(1-r) \omega+r \pi\left(p_{1}\right)-c\left(p_{1}\right)$, \\
Does not supervise & $(1-r)\left[\pi\left(p_{1}\right)-\omega\right]$ \\
\hline
\end{tabular}

breaks the contract under no supervision of the LSI). The expected payoff matrix of the LSI and FLSP $i$ is shown in Table 3.

The expected revenue function of the LSI is

$$
\begin{aligned}
\Pi_{I}^{2}(x, y) \\
=\Pi_{I}^{1}(x, y) \\
\quad-\{x[y+(1-y)(1-\rho)]+(1-x)[y+(1-y)]\} \alpha \\
=(A+\alpha \rho) x+(1-r) \Delta \pi y-(\theta+\alpha) \rho x y \\
\quad+(1-r) \pi\left(p_{2}\right)-(1-r) \omega-\alpha .
\end{aligned}
$$

We let $\partial \Pi_{I}^{2}(x, y) / \partial x=0$ to obtain

$$
y_{2}^{*}=\frac{A+\alpha \rho}{(\theta+\alpha) \rho} .
$$

The expected revenue function of FLSP $i$ is

$$
\begin{aligned}
\Pi_{F_{i}}^{2}(x, y) \\
=\Pi_{I}^{1}(x, y) \\
\quad+\{x[y+(1-y)(1-\rho)]+(1-x)[y+(1-y)]\} \alpha \\
=-(\theta+\alpha) \rho x+(T+\gamma N b R) y+(\theta+\alpha) \rho x y \\
\quad+(1-r) \omega+r \pi\left(p_{2}\right)-c\left(p_{2}\right)+\varphi+\alpha-\gamma N b R .
\end{aligned}
$$

We let $\partial \Pi_{F_{i}}^{2}(x, y) / \partial y=0$ to obtain

$$
x_{2}^{*}=\frac{-T-\gamma N b R}{(\theta+\alpha) \rho} .
$$

Comparing (6), (8), (10), and (12), we find that the introduction of compensation mechanism makes the LSI's optimal supervision probability decrease and FLSP $i$ 's optimal compliance probability increase.
FLSP

Breaks the contract

$(1-r) \omega+r \pi\left(p_{2}\right)-c\left(p_{2}\right)+\varphi-\rho \theta-\gamma N b R$,
$(1-r)\left[\pi\left(p_{2}\right)-\omega\right]-f(q)+\rho \theta$
$(1-r) \omega+r \pi\left(p_{2}\right)-c\left(p_{2}\right)+\varphi-\gamma N b R$,
$(1-r)\left[\pi\left(p_{2}\right)-\omega\right]$

Next, we will find out the range of the compensation value $\alpha$. Obviously, in order to obtain the LSI's optimal supervision probability, FLSP $i$ 's optimal compliance probability, and the expected revenue of the LSI and FLSP $i$ at the same time, (13) must be satisfied,

$$
\begin{aligned}
y_{2}^{*} & \geq y_{1}^{*}, \\
x_{2}^{*} & \leq x_{1}^{*}, \\
\Pi_{I}^{2}\left(x_{2}^{*}, y_{2}^{*}\right) & \geq \Pi_{I}^{1}\left(x_{1}^{*}, y_{1}^{*}\right), \\
\Pi_{F_{i}}^{2}\left(x_{2}^{*}, y_{2}^{*}\right) & \geq \Pi_{F_{i}}^{1}\left(x_{1}^{*}, y_{1}^{*}\right) .
\end{aligned}
$$

According to (6), (8), (10), and (12), the first two inequalities in (13) are always satisfied.

From the third inequality in (13), after calculation, we know that $\alpha \leq((1-r) \Delta \pi f(q) / \rho \theta)-\theta$. To make $\alpha \geq 0$, $(1-r) \Delta \pi f(q) / \rho \theta \geq \theta$ must be satisfied; that is, $(1-r) \Delta \pi f(q) \geq$ $\rho \theta^{2}$.

From the forth inequality in (13), after calculation we know that $\alpha \geq 0$.

In conclusion, the range of $\alpha$ is

$$
0 \leq \alpha \leq \frac{(1-r) \Delta \pi f(q)}{\rho \theta}-\theta .
$$

4.2. Design of Compensation Mechanism and the Optimal $N$. The compensation value $\alpha$ is in the interval of $[0,((1-$ $r) \Delta \pi f(q) / \rho \theta)-\theta$ ] according to Section 4.1. In practical applications, when implementing compensation mechanism, the LSI has to consider the specific methods of compensating FLSPs as well as the compensation value. In this section, we design three specific compensation mechanisms, that is, fixed compensation mechanism, linear compensation mechanism, and nonlinear compensation mechanism, and calculate their range of application, respectively.

4.2.1. Compensation Mechanism 1: Fixed Compensation Mechanism. The LSI compensates $\alpha=\alpha_{0}$ to FLSP $i$ when FLSP $i$ 
TABLE 3: Expected revenue matrix of LSI and FLSP $i$ with FLSPs' competition under a compensation mechanism.

\begin{tabular}{|c|c|c|}
\hline \multirow{2}{*}{ LSI } & \multicolumn{2}{|c|}{ FLSP } \\
\hline & Complies with the contract & Breaks the contract \\
\hline Supervises & $\begin{array}{l}(1-r) \omega+r \pi\left(p_{1}\right)-c\left(p_{1}\right)+\alpha \\
(1-r)\left[\pi\left(p_{1}\right)-\omega\right]-f(q)-\alpha\end{array}$ & $\begin{array}{l}(1-r) \omega+r \pi\left(p_{2}\right)-c\left(p_{2}\right)+\varphi-\rho \theta-\gamma N b R+(1-\rho) \alpha \\
(1-r)\left[\pi\left(p_{2}\right)-\omega\right]-f(q)+\rho \theta-(1-\rho) \alpha\end{array}$ \\
\hline Does not supervise & $\begin{array}{l}(1-r) \omega+r \pi\left(p_{1}\right)-c\left(p_{1}\right)+\alpha \\
(1-r)\left[\pi\left(p_{1}\right)-\omega\right]-\alpha\end{array}$ & $\begin{array}{l}(1-r) \omega+r \pi\left(p_{2}\right)-c\left(p_{2}\right)+\varphi-\gamma N b R+\alpha \\
(1-r)\left[\pi\left(p_{2}\right)-\omega\right]-\alpha\end{array}$ \\
\hline
\end{tabular}

complies with the contract (including the situation that FLSP $i$ breaks the contract but is not discovered and that FLSP $i$ breaks the contract without the LSI's supervision).

(1) To adopt compensation mechanism 1 , the number of FLSPs should meet

$$
0 \leq \alpha_{0} \leq \frac{(1-r) \Delta \pi f(q)}{\rho \theta}-\theta .
$$

Thus

$$
N \in Z \text {. }
$$

Proposition 6. As long as $0 \leq \alpha_{0} \leq((1-r) \Delta \pi f(q) / \rho \theta)-\theta$ is satisfied, the LSI can adopt fixed compensation mechanism, no matter how many FLSPs it has.

Proposition 6 shows that the LSI does not need to care about the number of FLSPs when it adopts the fixed compensation mechanism, as long as the compensation it pays to each FLSP is no more than $((1-r) \Delta \pi f(q) / \rho \theta)-\theta$.

(2) The impact of $N$ on the efficiency of the supply chain collaboration is as follows.

The optimal supervision probability of the LSI is

$$
\begin{aligned}
x_{2}^{* 1} & =\frac{-T-\gamma N b R}{(\theta+\alpha) \rho}=\frac{-T-\gamma N b R}{\left(\theta+\alpha_{0}\right) \rho}, \\
\frac{\partial x_{2}^{* 1}}{\partial N} & =-\frac{\gamma b R}{\left(\theta+\alpha_{0}\right) \rho}<0 .
\end{aligned}
$$

The optimal compliance probability of FLSP $i$ is

$$
\begin{aligned}
y_{2}^{* 1} & =\frac{A+\alpha \rho}{(\theta+\alpha) \rho}=\frac{A+\alpha_{0} \rho}{\left(\theta+\alpha_{0}\right) \rho}, \\
\frac{\partial y_{2}^{* 1}}{\partial N} & =0 .
\end{aligned}
$$

According to the analysis above, when using the fixed compensation mechanism, the LSI's optimal supervision probability is negatively correlated to $N$, while FLSP is optimal compliance probability is not related to $N$. Thus, Proposition 7 is obtained.

Proposition 7. A large $N$ will lead to high efficiency of the supply chain cooperation (a lower optimal supervision probability of the LSI and a higher optimal compliance probability of FLSP i). Thus, when adopting the fixed compensation mechanism, the efficiency of the supply chain cooperation reaches the maximum when $N=\infty$.
Propositions 6 and 7 show that the LSI can always adopt the fixed compensation mechanism no matter how many FLSPs it has, and the efficiency of the fixed compensation mechanism improves as $N$ increases.

4.2.2. Compensation Mechanism 2: Linear Compensation Mechanism. The LSI compensates $\alpha=\alpha_{1}+b_{1} N$ to FLSP $i$ when FLSP $i$ complies with the contract (including the situation that FLSP $i$ breaks the contract but is not discovered and that FLSP $i$ breaks the contract without the LSI's supervision).

(1) To adopt compensation mechanism 2, the number of FLSPs should meet

$$
0 \leq \alpha_{1}+b_{1} N \leq \frac{(1-r) \Delta \pi f(q)}{\rho \theta}-\theta .
$$

Thus,

$$
0 \leq N \leq \overline{N_{1}}
$$

in which $\overline{N_{1}}=\left((1-r) \Delta \pi f(q)-\rho \theta^{2}-\rho \theta \alpha_{1}\right) / \rho \theta b_{1}$.

Proposition 8. If $\overline{N_{1}}>0$, the LSI can adopt the linear compensation mechanism when the number of FLSPs satisfies $N \in\left(0, \overline{N_{1}}\right]$; otherwise, the LSI's optimal supervision probability, FLSP i's optimal compliance probability, and the expected revenue of the LSI and FLSP $i$ cannot be simultaneously optimized by using the linear compensation mechanism.

Proposition 8 shows that the linear compensation mechanism only applies to cases that the number of FLSPs is small, and it does not apply to those LSIs who have a large number of FLSPs.

In the following analysis, we assume that $\overline{N_{1}}>0$, which means that the LSI can adopt the linear compensation mechanism when $N \in\left(0, \overline{N_{1}}\right]$.

(2) The impact of $N$ on the efficiency of the supply chain collaboration is as follows.

The optimal supervision probability of the LSI is

$$
\begin{aligned}
x_{2}^{* 2} & =\frac{-T-\gamma N b R}{(\theta+\alpha) \rho}=\frac{-T-\gamma N b R}{\left[\theta+\left(\alpha_{1}+b_{1} N\right)\right] \rho}, \\
\frac{\partial x_{2}^{* 2}}{\partial N} & =\frac{b_{1} T-\left(\theta+\alpha_{1}\right) \gamma b R}{\left(\theta+\alpha_{1}+b_{1} N\right)^{2} \rho}<0 .
\end{aligned}
$$


The optimal compliance probability of FLSP $i$ is

$$
\begin{aligned}
y_{2}^{* 2} & =\frac{A+\alpha \rho}{(\theta+\alpha) \rho}=\frac{A+\left(\alpha_{1}+b_{1} N\right) \rho}{\left[\theta+\left(\alpha_{1}+b_{1} N\right)\right] \rho}, \\
\frac{\partial y_{2}^{* 2}}{\partial N} & =\frac{f(q) b_{1}}{\left(\theta+\alpha_{1}+b_{1} N\right)^{2} \rho}>0 .
\end{aligned}
$$

According to the analysis above, when using the linear compensation mechanism, the LSI's optimal supervision probability is negatively correlated to $N$, while FLSP is optimal compliance probability is positively correlated to $N$. Thus, Proposition 9 is obtained.

Proposition 9. A large $N$ will lead to high efficiency of the supply chain cooperation (a lower optimal supervision probability of the LSI and a higher optimal compliance probability of FLSP i). Thus, when adopting the linear compensation mechanism, the efficiency of the supply chain cooperation reaches the maximum when $N=\left\lfloor\overline{N_{1}}\right\rfloor$.

Propositions 8 and 9 show that the LSI can adopt the linear compensation mechanism when the number of FLSPs is small $\left(N \in\left(0, \overline{N_{1}}\right]\right)$, and the efficiency of the linear compensation mechanism achieves the maximum when $N=$ $\left\lfloor\overline{N_{1}}\right\rfloor$.

4.2.3. Compensation Mechanism 3: Nonlinear Compensation Mechanism. The LSI compensates $\alpha=\alpha_{2}+\left(b_{2} / N\right)$ to FLSP $i$ when FLSP $i$ complies with the contract (including the situation that FLSP $i$ breaks the contract but is not discovered and that FLSP $i$ breaks the contract without the LSI's supervision).

(1) To adopt compensation mechanism 3, the number of FLSPs should meet

$$
0 \leq \alpha_{2}+\frac{b_{2}}{N} \leq \frac{(1-r) \Delta \pi f(q)}{\rho \theta}-\theta .
$$

Thus

$$
N \geq \underline{N_{2}}
$$

in which $N_{2}=\rho \theta b_{2} /\left((1-r) \Delta \pi f(q)-\rho \theta^{2}-\rho \theta \alpha_{2}\right)$.

Proposition 10. If $\mathrm{N}_{2}>0$, the LSI can adopt the nonlinear compensation mechanism when the number of FLSPs satisfies $N \in\left[N_{2}, \infty\right)$; otherwise, the LSI can adopt the nonlinear compensation mechanism when the number of FLSPs is a positive integer.

Proposition 10 shows that the nonlinear compensation mechanism applies to cases that the number of FLSPs is large, and it may not apply to those LSIs who have a small number of FLSPs.

In the following analysis, we assume that $N_{2}>0$, which means that the LSI can adopt the nonlinear compensation mechanism when $N \in\left[N_{2}, \infty\right)$.

(2) The impact of $N \overline{\text { on }}$ the efficiency of the supply chain collaboration is as follows.
The optimal supervision probability of the LSI is

$$
x_{2}^{* 3}=\frac{-T-\gamma N b R}{(\theta+\alpha) \rho}=-\frac{N T+\gamma N^{2} b R}{\left(N \theta+N \alpha_{2}+b_{2}\right) \rho} \text {. }
$$

Since $\partial^{2} x_{2}^{* 3} / \partial N^{2}=-\left(2 \gamma b R b_{2}^{2}-2\left(\theta+\alpha_{2}\right) b_{2} T\right) /$ $\left(N \theta+N \alpha_{2}+b_{2}\right)^{3} \rho<0, x_{2}^{* 3}$ reaches the maximum when $\partial x_{2}^{* 3} / \partial N=0$; that is, $b_{2} T+\gamma N b R\left(N \theta+N \alpha_{2}+2 b_{2}\right)=0$.

The optimal compliance probability of FLSP $i$ is

$$
\begin{aligned}
y_{2}^{* 3} & =\frac{A+\alpha \rho}{(\theta+\alpha) \rho}=\frac{N A+\left(N \alpha_{2}+b_{2}\right) \rho}{\left(N \theta+N \alpha_{2}+b_{2}\right) \rho}, \\
\frac{\partial y_{2}^{* 3}}{\partial N} & =-\frac{f(q) b_{2}}{\left(N \theta+N \alpha_{2}+b_{2}\right)^{2} \rho}<0 .
\end{aligned}
$$

According to the analysis above, when using the nonlinear compensation mechanism, the LSI's optimal supervision probability is firstly positively and then negatively correlated to $N$, while FLSP is optimal compliance probability is negatively correlated to $N$. Thus, Proposition 11 is obtained.

Proposition 11. A small $N$ will lead to high efficiency of the supply chain cooperation (a lower optimal supervision probability of the LSI and a higher optimal compliance probability of FLSP i). Thus, when adopting the nonlinear compensation mechanism, the efficiency of the supply chain cooperation reaches the maximum when $N=\left\lceil N_{2}\right\rceil$.

Propositions 10 and 11 show that the LSI can adopt the nonlinear compensation mechanism when the number of FLSPs is large $\left(N \in\left[N_{2}, \infty\right)\right)$, and the efficiency of the nonlinear compensation mechanism achieves the maximum when $N=\left\lceil N_{2}\right\rceil$.

4.3. Comparative Analysis of Compensation Mechanisms. According to the analysis in Section 4.2, the three kinds of compensation mechanisms apply to different scopes. For LSIs who have different number of FLSPs, which mechanism is the most suitable one? This issue is discussed in this section.

4.3.1. The Conditions When Compensation Mechanism 1 (Fixed Compensation Mechanism) Is the Optimal Choice. When compensation mechanism 1 has the highest efficiency, (27) is satisfied:

$$
\begin{aligned}
& x_{2}^{* 1} \leq x_{2}^{* 2}, \\
& x_{2}^{* 1} \leq x_{2}^{* 3}, \\
& y_{2}^{* 1} \geq y_{2}^{* 2}, \\
& y_{2}^{* 1} \geq y_{2}^{* 3} .
\end{aligned}
$$


Therefore, when compensation mechanism 1 (fixed compensation mechanism) is the optimal choice, (28) is satisfied:

$$
\begin{cases}\frac{b_{2}}{\alpha_{0}-\alpha_{2}} \leq N \leq \frac{\alpha_{0}-\alpha_{1}}{b_{1}}, & \text { if }\left(\alpha_{0}>\alpha_{2}\right) \cap\left(\alpha_{0}>\alpha_{1}\right) \cap \\ & \left(\frac{b_{2}}{\alpha_{0}-\alpha_{2}} \leq \frac{\alpha_{0}-\alpha_{1}}{b_{1}}\right) \\ \emptyset, & \text { else. }\end{cases}
$$

4.3.2. The Conditions When Compensation Mechanism 2 (Linear Compensation Mechanism) Is the Optimal Choice. When compensation mechanism 2 has the highest efficiency, (29) is satisfied:

$$
\begin{aligned}
& x_{2}^{* 2} \leq x_{2}^{* 1}, \\
& x_{2}^{* 2} \leq x_{2}^{* 3}, \\
& y_{2}^{* 2} \geq y_{2}^{* 1}, \\
& y_{2}^{* 2} \geq y_{2}^{* 3} .
\end{aligned}
$$

Equation (29) could be calculated and changed into

$$
N \geq\left\{\begin{array}{c}
\frac{\alpha_{0}-\alpha_{1}}{b_{1}} \\
N_{4},
\end{array}\right.
$$

in which it is assumed that $N_{3}=\left(-\left(\alpha_{1}-\alpha_{2}\right)-\right.$ $\left.\sqrt{\left(\alpha_{1}-\alpha_{2}\right)^{2}+4 b_{1} b_{2}}\right) / 2 b_{1}<0, N_{4}=\left(-\left(\alpha_{1}-\alpha_{2}\right)+\right.$ $\sqrt{\left.\left(\alpha_{1}-\alpha_{2}\right)^{2}+4 b_{1} b_{2}\right)} / 2 b_{1}>0$.

Therefore, when compensation mechanism 2 (linear compensation mechanism) is the optimal choice, (31) is satisfied:

$$
N \geq \begin{cases}\frac{\alpha_{0}-\alpha_{1}}{b_{1}}, & \text { if } \frac{\alpha_{0}-\alpha_{1}}{b_{1}}>N_{4}, \\ N_{4}, & \text { if } \frac{\alpha_{0}-\alpha_{1}}{b_{1}} \leq N_{4} .\end{cases}
$$

When adopting linear compensation mechanism, there is an upper limit $\overline{N_{1}}$ for the number of FLSPs. In this paper, we assume $\overline{N_{1}} \geq N_{4}$ and $\overline{N_{1}} \geq\left(\alpha_{0}-\alpha_{1}\right) / b_{1}$ to ensure that the linear compensation mechanism can take every value in the optimal interval.

4.3.3. The Conditions When Compensation Mechanism 3 (Nonlinear Compensation Mechanism) Is the Optimal Choice. When compensation mechanism 3 has the highest efficiency, (32) is satisfied:

$$
\begin{aligned}
& x_{2}^{* 3} \leq x_{2}^{* 1}, \\
& x_{2}^{* 3} \leq x_{2}^{* 2}, \\
& y_{2}^{* 3} \geq y_{2}^{* 1}, \\
& y_{2}^{* 3} \geq y_{2}^{* 2} .
\end{aligned}
$$

Therefore, when compensation mechanism 3 (nonlinear compensation mechanism) is the optimal choice, the nether inequalities are satisfied:

$$
0<N \leq \begin{cases}\frac{b_{2}}{\alpha_{0}-\alpha_{2}}, & \left(\alpha_{0}>\alpha_{2}\right) \cap\left(N_{4} \geq \frac{b_{2}}{\alpha_{0}-\alpha_{2}}\right), \\ N_{4}, & \left(\alpha_{0} \leq \alpha_{2}\right) \\ & \cup\left(\alpha_{0}>\alpha_{2} \cap N_{4}<\frac{b_{2}}{\alpha_{0}-\alpha_{2}}\right) .\end{cases}
$$

When adopting nonlinear compensation mechanism, there is a lower limit $N_{2}$ for the number of FLSPs. In this paper, we assume $N_{2} \leq N_{4}$ and $N_{2} \leq b_{2} /\left(\alpha_{0}-\alpha_{2}\right)$ to ensure that the nonlinear compensation mechanism can take every value in the optimal interval.

Therefore, when compensation mechanism 3 (nonlinear compensation mechanism) is the optimal choice, (34) is satisfied:

$$
\underline{N_{2}}<N \leq \begin{cases}\frac{b_{2}}{\alpha_{0}-\alpha_{2}}, & \left(\alpha_{0}>\alpha_{2}\right) \cap\left(N_{4} \geq \frac{b_{2}}{\alpha_{0}-\alpha_{2}}\right), \\ N_{4}, & \left(\alpha_{0} \leq \alpha_{2}\right) \\ & \cup\left(\alpha_{0}>\alpha_{2} \cap N_{4}<\frac{b_{2}}{\alpha_{0}-\alpha_{2}}\right) .\end{cases}
$$

4.3.4. The Optimal Compensation Mechanism with Different Number of FLSPS. According to the analysis in Sections 4.3.1 to 4.3.3, the optimal compensation mechanism with different number of FLSPs is shown in Table 4.

Table 4 shows that LSIs should follow the principles below.

(1) If $\alpha_{0} \leq \alpha_{2}$, the LSI should adopt the nonlinear compensation mechanism when $N \in\left(0, N_{4}\right]$.

(2) If $\alpha_{0}>\alpha_{2}$, the LSI should adopt the fixed compensation mechanism when $N \in\left[b_{2} /\left(\alpha_{0}-\alpha_{2}\right),\left(\alpha_{0}-\alpha_{1}\right) / b_{1}\right]$ (if $b_{2} /\left(\alpha_{0}-\alpha_{2}\right) \leq\left(\alpha_{0}-\alpha_{1}\right) / b_{1}$ and $\left.\alpha_{0}>\alpha_{1}\right)$ and adopt the nonlinear compensation mechanism when $N \in\left[N_{2}, b_{2} /\left(\alpha_{0}-\alpha_{2}\right)\right]$ (if $N_{4} \geq b_{2} /\left(\alpha_{0}-\alpha_{2}\right)$ ) or $N \in\left[\overline{N_{2}, N_{4}}\right]$ (if $\left.N_{4}<b_{2} /\left(\alpha_{0}-\alpha_{2}\right)\right)$.

(3) The LSI should adopt the linear compensation mechanism when $N \in\left[\left(\alpha_{0}-\alpha_{1}\right) / b_{1}, \overline{N_{1}}\right]$ (if $N_{4}<\left(\alpha_{0}-\right.$ $\left.\alpha_{1}\right) / b_{1}$ ) or $N \in\left[N_{4}, \overline{N_{1}}\right]$ (if $\left.N_{4} \geq\left(\alpha_{0}-\alpha_{1}\right) / b_{1}\right)$.

The fixed compensation mechanism is never the optimal choice when $\alpha_{0} \leq \alpha_{1}$ or $\alpha_{0} \leq \alpha_{2}$. Therefore, we assume $\alpha_{0}>$ $\alpha_{1}$ and $\alpha_{0}>\alpha_{2}$ in the following analysis. Moreover, we assume $\overline{N_{1}} \geq b_{2} /\left(\alpha_{0}-\alpha_{2}\right)$ and $N_{2} \leq\left(\alpha_{0}-\alpha_{1}\right) / b_{1}$ to simplify the analysis.

Since the size relation of $N_{4},\left(\alpha_{0}-\alpha_{1}\right) / b_{1}$ and $b_{2} /\left(\alpha_{0}-\alpha_{2}\right)$ remains uncertain, there are six possible relations according to the Permutations and Combinations Theory. Their size relation influences the optimal intervals of the three compensation mechanisms. Thus, the optimal compensation mechanism under different intervals of $N$ is shown in Figures $2,3,4,5,6$, and 7 . 
TABLE 4: The optimal compensation mechanism with different number of FLSPs.

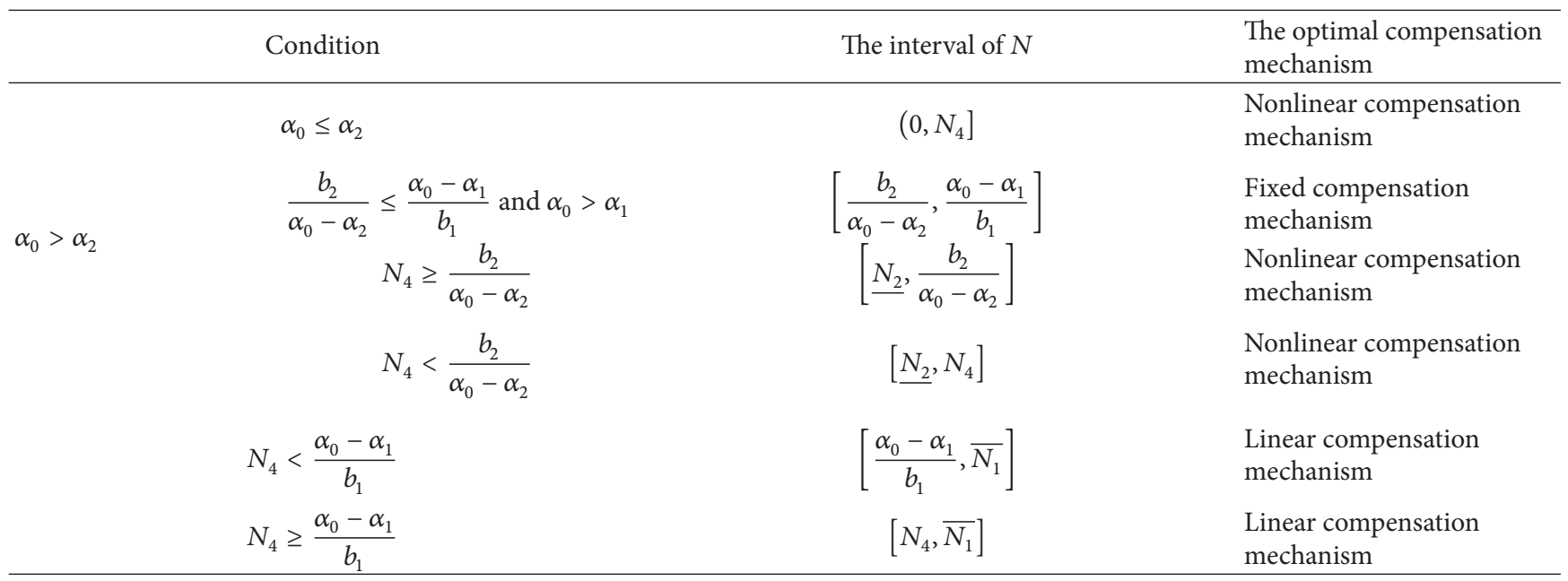

The following three conclusions can be obtained from Figures 2-7.

(1) The LSI should adopt nonlinear compensation mechanism when the number of FLSPs is small ( $N \leq N_{4}$ and $\left.N \leq b_{2} /\left(\alpha_{0}-\alpha_{2}\right)\right)$. This suggests that those LSIs who ask for customized or upscale services from FLSPs should give priority to nonlinear compensation mechanism. However, if the number of FLSPs is smaller than the lower limit $N_{2}$, the LSI can only select from the fixed compensation mechanism and the linear compensation mechanism.

(2) The LSI should adopt linear compensation mechanism when the number of FLSPs is large $\left(N \geq N_{4}\right.$ and $\left.N \geq\left(\alpha_{0}-\alpha_{1}\right) / b_{1}\right)$. This suggests that those LSIs who ask for mass or humble services from FLSPs should give priority to linear compensation mechanism. However, if the number of FLSPs is larger than the higher limit $\overline{N_{1}}$, the LSI can only select from the fixed compensation mechanism and the nonlinear compensation mechanism.

(3) It can be seen from Figures 2-7 that there is no optimal compensation mechanism when the number of FLSPs is in other intervals. This is because in these cases, the optimal supervision probability of the LSI and the optimal compliance probability of FLSPs cannot be optimized at the same time. Thus, the LSI has to balance the importance of these two variables in the selection of these compensation mechanisms.

\section{Numerical Analysis}

Section 5 conducts numerical analysis with MATLAB 8.0 software, which verifies the propositions provided above.

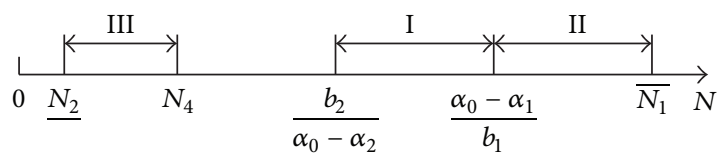

FIgURE 2: The optimal compensation mechanism in different intervals when $N_{4}<b_{2} /\left(\alpha_{0}-\alpha_{2}\right)<\left(\alpha_{0}-\alpha_{1}\right) / b_{1}$.

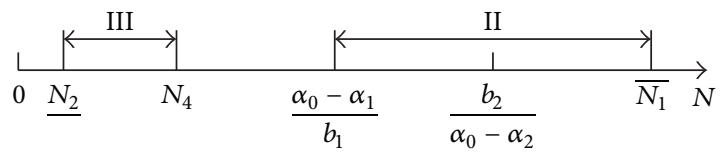

FIGURE 3: The optimal compensation mechanism in different intervals when $N_{4}<\left(\alpha_{0}-\alpha_{1}\right) / b_{1}<b_{2} /\left(\alpha_{0}-\alpha_{2}\right)$.

Some of the parameters involved in the numerical analysis are as follows:

$$
\begin{aligned}
\omega=25, & \rho=0.6, \\
\theta=40, \quad f(q)=22, \quad & \quad r=0.3, \\
\pi\left(p_{1}\right)=175, & \quad c\left(p_{2}\right)=80, \\
c\left(p_{1}\right)=25, & \gamma=0.2, \\
\varphi=38, & R=40 ; \\
b=0.2, & \alpha_{1}=10,
\end{aligned}
$$




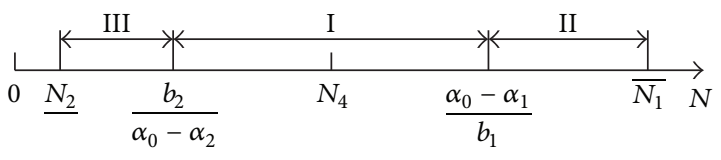

FIgURE 4: The optimal compensation mechanism in different intervals when $b_{2} /\left(\alpha_{0}-\alpha_{2}\right)<N_{4}<\left(\alpha_{0}-\alpha_{1}\right) / b_{1}$.

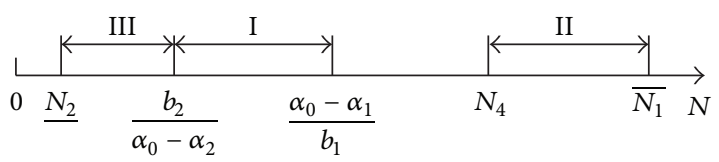

FIGURE 5: The optimal compensation mechanism in different intervals when $b_{2} /\left(\alpha_{0}-\alpha_{2}\right)<\left(\alpha_{0}-\alpha_{1}\right) / b_{1}<N_{4}$.

$$
\begin{gathered}
b_{1}=4, \quad \alpha_{2}=20, \quad b_{2}=40 ; \\
\Delta \pi=\pi\left(p_{1}\right)-\pi\left(p_{2}\right)=95, \\
A=\rho \theta-f(q)=2, \\
T=r \Delta \pi-\left[c\left(p_{1}\right)-c\left(p_{2}\right)\right]-\varphi=-24.5 .
\end{gathered}
$$

Substituting the values of some of the parameters provided above into (17), (18), (21), (22), (25), and (26), the changes of $x_{2}^{*}$ and $y_{2}^{*}$ as $N$ changes can be obtained, as shown in Figures 8, 9, and 10.

$x_{2}^{* 1}<x_{2}^{* 2}$ at the left side of $A(3.75,0.47)$, which means that compensation mechanism 1 is better than compensation mechanism $2 ; x_{2}^{* 1}>x_{2}^{* 2}$ at the right side of $A$, which means compensation mechanism 2 is better than compensation mechanism 1. $y_{2}^{* 1}>y_{2}^{* 2}$ at the left side of $B(3.75,0.44)$, which means compensation mechanism 1 is better than compensation mechanism 2; $y_{2}^{* 1}<y_{2}^{* 2}$ at the right side of $B$, which means compensation mechanism 2 is better than compensation mechanism 1. Consequently, compensation mechanism 1 is better than compensation mechanism 2 when $N \in(0,3.75]$, and compensation mechanism 2 is better than compensation mechanism 1 when $N \in[3.75,12]$.

$x_{2}^{* 1}>x_{2}^{* 3}$ at the left side of $C(8,0.3)$, which means compensation mechanism 3 is better than compensation mechanism $1 ; x_{2}^{* 1}<x_{2}^{* 3}$ at the right side of $C$, which means compensation mechanism 1 is better than compensation mechanism 3. $y_{2}^{* 1}<y_{2}^{* 3}$ at the left side of $D(8,0.44)$, which means compensation mechanism 3 is better than compensation mechanism $1 ; y_{2}^{* 1}>y_{2}^{* 3}$ at the right side of $D$, which means compensation mechanism 1 is better than compensation mechanism 3. Consequently, compensation mechanism 3 is better than compensation mechanism 1 when $N \in(0,8]$, and compensation mechanism 1 is better than compensation mechanism 3 when $N \in[8,12]$.

$x_{2}^{* 2}>x_{2}^{* 3}$ at the left side of $E(4.65,0.41)$, which means compensation mechanism 3 is better than compensation mechanism $2 ; x_{2}^{* 2}<x_{2}^{* 3}$ at the right side of $E$, which means compensation mechanism 2 is better than compensation

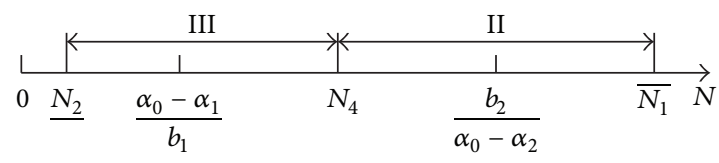

FIGURE 6: The optimal compensation mechanism in different intervals when $\left(\alpha_{0}-\alpha_{1}\right) / b_{1}<N_{4}<b_{2} /\left(\alpha_{0}-\alpha_{2}\right)$.

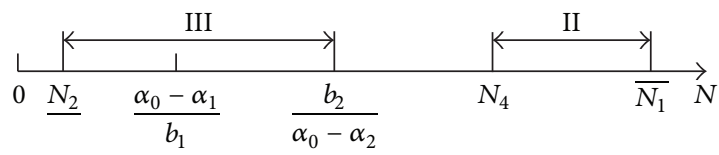

FIgURE 7: The optimal compensation mechanism in different intervals when $\left(\alpha_{0}-\alpha_{1}\right) / b_{1}<b_{2} /\left(\alpha_{0}-\alpha_{2}\right)<N_{4}$.

mechanism 3. $y_{2}^{* 2}<y_{2}^{* 3}$ at the left side of $F(4.65,0.47)$, which means compensation mechanism 3 is better than compensation mechanism $2 ; y_{2}^{* 2}>y_{2}^{* 3}$ at the right side of $F$, which means compensation mechanism 2 is better than compensation mechanism 3. Consequently, compensation mechanism 3 is better than compensation mechanism 2 when $N \in(0,4.65]$, and compensation mechanism 2 is better than compensation mechanism 3 when $N \in[4.65,12]$.

In conclusion, compensation mechanism 1 is better than compensation mechanism 2 when $N \in(0,3.75]$, and compensation mechanism 2 is better than compensation mechanism 1 when $N \in[3.75,12]$; compensation mechanism 3 is better than compensation mechanism 1 when $N \in(0,8]$, and compensation mechanism 1 is better than compensation mechanism 3 when $N \in[8,12]$; compensation mechanism 3 is better than compensation mechanism 2 when $N \in$ $(0,4.65]$, and compensation mechanism 2 is better than compensation mechanism 3 when $N \in[4.65,12]$.

In consequence, compensation mechanism 2 is better than compensation mechanisms 1 and 3 when $N \in[4.65,12]$, compensation mechanism 3 is better than compensation mechanisms 1 and 2 when $N \in(0,4.65]$, and compensation mechanism 1 is not the best choice in any interval. (According to the analysis in Section 4.3.4, if $\alpha_{0}>\alpha_{2}$, the LSI should adopt the fixed compensation mechanism when $N \in$ $\left[b_{2} /\left(\alpha_{0}-\alpha_{2}\right),\left(\alpha_{0}-\alpha_{1}\right) / b_{1}\right]\left(\right.$ if $b_{2} /\left(\alpha_{0}-\alpha_{2}\right) \leq\left(\alpha_{0}-\alpha_{1}\right) / b_{1}$ and $\left.\alpha_{0}>\alpha_{1}\right)$, while in this section, we assume $b_{2} /\left(\alpha_{0}-\alpha_{2}\right)=8$ and $\left(\alpha_{0}-\alpha_{1}\right) / b_{1}=3.75$, which leads to $b_{2} /\left(\alpha_{0}-\alpha_{2}\right)>\left(\alpha_{0}-\alpha_{1}\right) / b_{1}$, so compensation mechanism 1 is not the best choice in any interval.) In other words, the LSI should adopt the nonlinear compensation mechanism when the number of FLSPs ranges from 1 to 4 and adopt the linear compensation mechanism when the number of FLSPs ranges from 5 to 12.

\section{Main Conclusions and Management Implications}

6.1. Main Conclusions. This paper studies the impact of the number of competitors on the effect of quality supervision game in a supply chain and finds that the original mixed payment contract cannot optimize the LSI's optimal supervision probability and FLSP is optimal compliance 


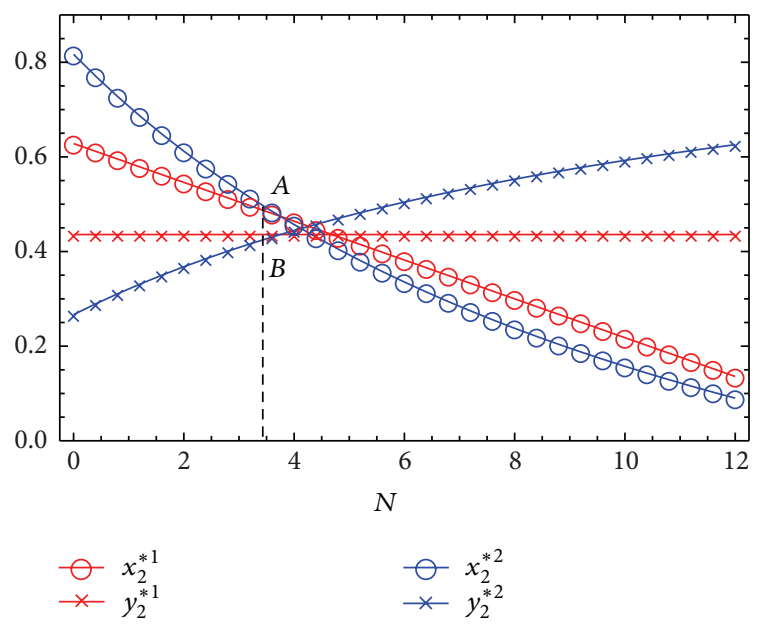

FIGURE 8: The changes of $x_{2}^{*}$ and $y_{2}^{*}$ as $N$ changes in compensation mechanisms 1 and 2.

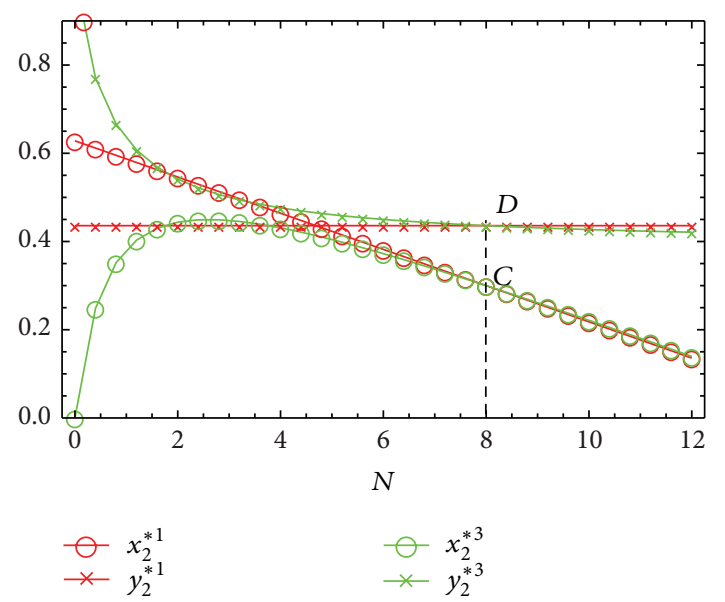

FIGURE 9: The changes of $x_{2}^{*}$ and $y_{2}^{*}$ as $N$ changes in compensation mechanisms 1 and 3.

probability at the same time. Therefore, the compensation mechanism is introduced into our model and it is proved that the compensation mechanism can lead to Pareto optimality. Based on this, the application conditions of three different compensation mechanisms are discussed, and it is proved that the compensation mechanism choice of the LSI is influenced by the number of FLSPs.

Firstly, the LSI should adopt nonlinear compensation mechanism when the number of FLSPs is small $\left(N \leq N_{4}\right.$ and $\left.N \leq b_{2} /\left(\alpha_{0}-\alpha_{2}\right)\right)$, but if the number of FLSPs is smaller than the lower limit $N_{2}$, LSI can only select from the fixed compensation mechanism and the linear compensation mechanism.

Secondly, LSI should adopt linear compensation mechanism when the number of FLSPs is large $\left(N \geq N_{4}\right.$ and $\left.N \geq\left(\alpha_{0}-\alpha_{1}\right) / b_{1}\right)$, but if the number of FLSPs is larger than the higher limit $\overline{N_{1}}$, LSI can only select from the fixed compensation mechanism and the nonlinear compensation mechanism.

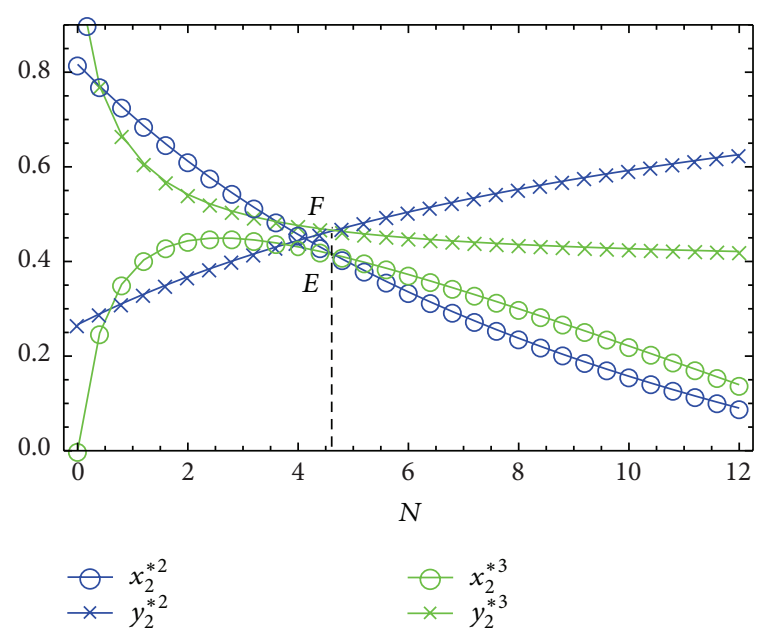

FIGURE 10: The changes of $x_{2}^{*}$ and $y_{2}^{*}$ as $N$ changes in compensation mechanisms 2 and 3.

Thirdly, there is no optimal compensation mechanism when the number of FLSPs is in other intervals, so LSI has to balance the importance of two variables, which are the LSI's optimal supervision probability and FLSP is optimal compliance probability, in the selection of compensation mechanism.

6.2. Management Implications. In this paper, we use game theory to obtain the mixed strategy Nash equilibrium of the quality supervision game, study the impact of the number of FLSPs on the quality supervision game in LSSC, and obtain applicable intervals for different compensation mechanisms. Our research provides a new idea for further studies on LSSC quality supervision game with competition among FLSPs. For instance, researchers can further explore, in addition to compensation mechanism, that other kinds of mechanism exist to improve the effectiveness of the quality supervision game in the supply chain. Also, researchers can further study on how to express the competitive relations and improve the effectiveness of the quality supervision game if the competitors are heterogeneous. In addition, this study provides a necessary theoretical basis for future empirical studies on the impact of the number of FLSPs on the quality supervision game of LSSC.

From the manager's perspective, our conclusions have significance in improving the effectiveness of quality supervision game in LSSC. LSIs, as the leader in the supply chain, can consider using compensation mechanisms to improve the effectiveness of quality supervision game when they have multiple competing FLSPs. LSIs that require different types of service need different numbers of FLSPs; correspondingly, the compensation mechanisms they should adopt are different. For example, the LSI who asks for customized or upscale services from a small number of FLSPs $\left(N \leq N_{4}\right.$ and $\left.N \leq b_{2} /\left(\alpha_{0}-\alpha_{2}\right)\right)$ should give priority to the nonlinear compensation mechanism; however, if the number of FLSPs is smaller than the lower limit $N_{2}$, the LSI can only select from the fixed compensation mechanism and the linear 
compensation mechanism. The LSI who asks for mass or humble services from a large number of FLSPs $\left(N \geq N_{4}\right.$ and $\left.N \geq\left(\alpha_{0}-\alpha_{1}\right) / b_{1}\right)$ should give priority to linear compensation mechanism; however, if the number of FLSPs is larger than the higher limit $\overline{N_{1}}$, the LSI can only select from the fixed compensation mechanism and the nonlinear compensation mechanism.

Furthermore, there is no optimal compensation mechanism in some cases. This is because in these cases, the optimal supervision probability of the LSI and the optimal compliance probability of FLSP $i$ cannot be optimized at the same time. Thus, the LSI has to balance the importance of these two variables in the selection of compensation mechanism.

This paper attempts to analyze the impact of the number of FLSPs on the quality supervision game of LSSC and provide a theoretical reference for the study on the quality supervision game. There are still some limitations worth to be improved in future studies. For instance, we assume that $\gamma, b$, and $R$ are the same for all FLSPs, but they may not be exactly the same in practice. In addition, the follow-up study might concentrate on empirical research to explore the impact of the number of FLSPs on the quality supervision game of LSSC in the reality.

\section{Appendix}

\section{Notations for the Model}

$p_{i}$ : Effort level of the FLSP: $i=1$ is the FLSP's effort level when it complies with the contract; $i=2$ is the FLSP's effort level when it does not comply with the LSI.

$\omega$ : Fixed payment that the FSLP gains from the LSI.

$r$ : Coefficient of revenue that the FLSP obtains from cooperation.

$c\left(p_{i}\right)$ : Cost of the FLSP's service.

$\pi\left(p_{i}\right)$ : Total revenue of the LSSC, which is a function of the FLSP's effort level, $\pi(p)=g(p)+\varepsilon$, in which $g(p)$ is a function of $p$.

$\varepsilon$ : External environment's impact on the total revenue of the LSSC, $\varepsilon \sim N\left(0, \sigma_{\varepsilon}^{2}\right)$.

$f(q)$ : Cost of the LSI when it supervises the FLSP's effort level, $f(q)=h(q)+\eta$, where $h(q)$ is a function of $q$ and $q$ is the supervision effort level of the LSI.

$\eta$ : External environment's impact on the supervision cost of the LSI, $\eta \sim N\left(0, \sigma_{\eta}^{2}\right)$.

$\varphi$ : Personal interests that the FLSP gains when it opts not to comply with the contract.

$\rho$ : Probability that the FLSP's noncompliance with the contract is discovered when the LSI supervises the FLSP's service quality: $\rho$ represents the measurability of the service quality; $\rho$ increases as the difficulty level of measuring service quality decreases.

$\theta$ : Penalty that the FLSP takes when it breaks the contract and is discovered by the LSI. $x$ : Supervision probability of the LSI: $(1-x)$ is the nonsupervision probability of the LSI.

$y$ : Compliance probability of the FLSP: $(1-y)$ is the noncompliance probability of the FLSP.

$\Pi_{I}^{0}(x, y)$ : The expected revenue of the LSI in the quality supervision game model for the LSSC without FLSP' competition: it is a function of $x$ and $y$.

$\Pi_{F}^{0}(x, y)$ : The expected revenue of the FLSP in the quality supervision game model for the LSSC without FLSP' competition: it is a function of $x$ and $y$.

$\Pi_{I}^{1}(x, y)$ : The expected revenue of the LSI in the quality supervision game model for the LSSC with FLSP' competition: it is a function of $x$ and $y$.

$\Pi_{F_{i}}^{1}(x, y)$ : The expected revenue of FLSP $i$ in the quality supervision game model for the LSSC with FLSP' competition: it is a function of $x$ and $y$.

$F_{i}(N)$ : The expected loss of FLSP $i$ due to the competition among FLSPs. $F_{i}(N)=\gamma N b R$.

$\gamma$ : Competitive impact coefficient.

$N$ : The number of FLSPs, $N \geq 2$.

$b$ : Competitive coefficient, that is, the degree of mutual influence among FLSPs, and $0<b<1$ : a large $b$ means that the business competition among FLSPs is fierce; in particular, there is not any mutual effect among FLSPs when $b=0$.

$R$ : The service quality level of the FLSP, which can be informed from the FLSP's past experience of cooperation or credibility: it is assumed in this paper that all the FLSPs are homogeneous; that is, their service qualities are at the same level.

$\Pi_{I}^{2}(x, y)$ : The expected revenue of the LSI in the quality supervision game model with FLSPs' competition under a compensation mechanism: it is a function of $x$ and $y$.

$\Pi_{F_{i}}^{2}(x, y)$ : The expected revenue of FLSP $i$ in the quality supervision game model with FLSPs' competition under a compensation mechanism: it is a function of $x$ and $y$.

$\alpha$ : The compensation that the LSI pays to FLSP $i$ when FLSP $i$ complies with the contract (including that FLSP $i$ breaks the contract but is not discovered and that FLSP $i$ breaks the contract under no supervision of the LSI): $\alpha>0$.

$\Delta \pi: \Delta \pi=\pi\left(p_{1}\right)-\pi\left(p_{2}\right)$ presents the difference between the total revenues of the LSSC when the FLSP adopts the strategy of compliance and noncompliance.

A: $A=\rho \theta-f(q)$ is equal to the net income of the LSI when it has supervision cost and penalty income; we can obtain that $0 \leq A \leq \rho \theta$ from (2) if $y^{*} \geq 0$.

$T: T=r \Delta \pi-\left[c\left(p_{1}\right)-c\left(p_{2}\right)\right]-\varphi:$ if $T>0$, then $r \pi\left(p_{1}\right)-$ $c\left(p_{1}\right)>r \pi\left(p_{2}\right)-c\left(p_{2}\right)+\varphi$, which means that, without considering the LSI's fixed payment and penalty to the 
FLSP, the total revenue when the FLSP complies with the contract is larger than that when the FLSP breaks the contract. On the contrary, $T<0$ means that, without considering the LSI's fixed payment and penalty to the FLSP, the total revenue when the FLSP complies with the contract is smaller than that when the FLSP breaks the contract. We can obtain that $0 \leq-T \leq$ $\rho \theta$ from (4).

$M: M=T+\gamma N b R:$ we can obtain that $-T-\gamma N b R \geq 0$ from (8).

\section{Conflict of Interests}

The authors declare that there is no conflict of interests regarding the publication of this paper.

\section{Acknowledgments}

This research is supported by the National Natural Science Foundation of China (Grant no. 71372156), supported by Humanity and Social Science Youth Foundation of Ministry of Education of China (Grant no. 2013YJC630098), and sponsored by China State Scholarship Fund and Independent Innovation Foundation of Tianjin University.

\section{References}

[1] L. Wei-hua, X. Xue-cai, R. Zheng-xu, and P. Yan, "An emergency order allocation model based on multi-provider in two-echelon logistics service supply chain," Supply Chain Management, vol. 16, no. 6, pp. 391-400, 2011.

[2] W. H. Liu, D. Xie, and X. Xu, "Quality supervision and coordination of logistic service supply chain under multi-period conditions," International Journal of Production Economics, vol. 142, no. 2, pp. 353-361, 2012.

[3] J. Y. Bakos and E. Brynjolfsson, "Information technology, incentives, and the optimal number of suppliers," Journal of Management Information Systems, vol. 10, no. 2, pp. 37-53, 1993.

[4] S. Y. Chen and M. H. Xie, "Third party logistics enterprise resource integration," China Logistics \& Purchasing, vol. 19, pp. 62-64, 2005.

[5] W. H. Liu and D. Xie, "Quality decision of the logistics service supply chain with service quality guarantee," International Journal of Production Research, vol. 51, no. 5, pp. 1618-1634, 2013.

[6] K. R. Balachandran and S. Radhakrishnan, "Quality implications of warranties in a supply chain," Management Science, vol. 51, no. 8, pp. 1266-1277, 2005.

[7] N. Seth, S. G. Deshmukh, and P. Vrat, "A framework for measurement of quality of service in supply chains," Supply Chain Management, vol. 11, no. 1, pp. 82-94, 2006.

[8] S. Baiman, P. E. Fischer, and M. V. Rajan, "Information, contracting, and quality costs," Management Science, vol. 46, no. 6, pp. 776-789, 2000.

[9] W. S. Lim, "Producer-supplier contracts with incomplete information," Management Science, vol. 47, no. 5, pp. 709-715, 2001.

[10] M. Kaya and Ö. Özer, "Quality risk in outsourcing: noncontractible product quality and private quality cost information," Naval Research Logistics, vol. 56, no. 7, pp. 669-685, 2009.
[11] G. H. Chao, S. M. R. Iravani, and R. C. Savaskan, "Quality improvement incentives and product recall cost sharing contracts," Management Science, vol. 55, no. 7, pp. 1122-1138, 2009.

[12] S. Hertz and M. Macquet, "How Third party logistics providers create effectiveness and efficiency by coordinating customers' activities an strategies," Stockholm School of Economics, no. 2011:10, 2001.

[13] J. Jayaram and K.-C. Tan, "Supply chain integration with thirdparty logistics providers," International Journal of Production Economics, vol. 125, no. 2, pp. 262-271, 2010.

[14] P. Kelle and E. A. Silver, "Decreasing expected shortages through order splitting," Engineering Costs and Production Economics, vol. 19, no. 1-3, pp. 351-357, 1990.

[15] W. D. Presutti, "The single source issue: US and Japanese sourcing strategies," International Journal of Purchasing and Materials Management, vol. 28, no. 1, pp. 2-9, 1992.

[16] R. V. Ramasesh, J. K. Ord, J. C. Hayya, and A. Pan, "Sole versus dual sourcing in stochastic lead time (s, Q) inventory models," Management Science, vol. 37, no. 4, pp. 428-443, 1991.

[17] P. D. Berger, A. Gerstenfeld, and A. Z. Zeng, "How many suppliers are best? A decision-analysis approach," Omega, vol. 32, no. 1, pp. 9-15, 2004.

[18] A. J. Ruiz-Torres and F. Mahmoodi, "The optimal number of suppliers considering the costs of individual supplier failures," Omega, vol. 35, no. 1, pp. 104-115, 2007.

[19] H. Yu, A. Z. Zeng, and L. Zhao, "Single or dual sourcing: decision-making in the presence of supply chain disruption risks," Omega, vol. 37, no. 4, pp. 788-800, 2009.

[20] S.-H. Nam, J. Vitton, and H. Kurata, "Robust supply base management: determining the optimal number of suppliers utilized by contractors," International Journal of Production Economics, vol. 134, no. 2, pp. 333-343, 2011.

[21] E. A. Demirtas and Ö. Üstün, "An integrated multiobjective decision making process for supplier selection and order allocation," Omega, vol. 36, no. 1, pp. 76-90, 2008.

[22] F. Faez, S. H. Ghodsypour, and C. O’Brien, "Vendor selection and order allocation using an integrated fuzzy case-based reasoning and mathematical programming model," International Journal of Production Economics, vol. 121, no. 2, pp. 395-408, 2009.

[23] A. S. Erdem and E. Göen, "Development of a decision support system for supplier evaluation and order allocation," Expert Systems with Applications, vol. 39, no. 5, pp. 4927-4937, 2012.

[24] B. Feng, Z.-P. Fan, and Y. Li, "A decision method for supplier selection in multi-service outsourcing," International Journal of Production Economics, vol. 132, no. 2, pp. 240-250, 2011.

[25] B. Feng, "Multisourcing suppliers selection in service outsourcing," Journal of the Operational Research Society, vol. 63, no. 5, pp. 582-596, 2012.

[26] C. J. Corbett, "Stochastic inventory systems in a supply chain with asymmetric information: cycle stocks, safety stocks, and consignment stock," Operations Research, vol. 49, no. 4, pp. 487500, 2001.

[27] H. Krishnan, R. Kapuscinski, and D. A. Butz, "Coordinating contracts for decentralized supply chains with retailer promotional effort," Management Science, vol. 50, no. 1, pp. 48-63, 2004.

[28] M. Ferguson, V. D. R. Guide Jr., and G. C. Souza, "Supply chain coordination for false failure returns," Manufacturing and Service Operations Management, vol. 8, no. 4, pp. 376-393, 2006. 
[29] T. A. Taylor, "Supply chain coordination under channel rebates with sales effort effects," Management Science, vol. 48, no. 8, pp. 992-1007, 2002.

[30] W. K. Wong, J. Qi, and S. Y. S. Leung, "Coordinating supply chains with sales rebate contracts and vendor-managed inventory," International Journal of Production Economics, vol. 120, no. 1, pp. 151-161, 2009.

[31] K. Chen and T. Xiao, "Demand disruption and coordination of the supply chain with a dominant retailer," European Journal of Operational Research, vol. 197, no. 1, pp. 225-234, 2009.

[32] C.-H. Chiu, T.-M. Choi, and C. S. Tang, "Price, rebate, and returns supply contracts for coordinating supply chains with price-dependent demands," Production and Operations Management, vol. 20, no. 1, pp. 81-91, 2011.

[33] S. A. Starbird, "Penalties, rewards, and inspection: povisions for quality in supply chain contracts," Journal of the Operational Research Society, vol. 52, no. 1, pp. 109-115, 2001.

[34] L. Zhang, S. Song, and C. Wu, "Supply chain coordination of loss-averse newsvendor with contract," Tsinghua Science and Technology, vol. 10, no. 2, pp. 133-140, 2005.

[35] L. F. Pitt, R. T. Watson, and C. B. Kavan, "Service quality: a measure of information systems effectiveness," MIS Quarterly, vol. 19, no. 2, pp. 173-185, 1995. 


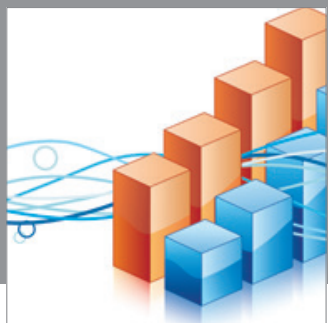

Advances in

Operations Research

mansans

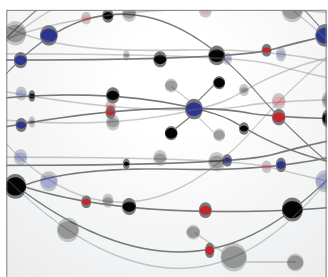

The Scientific World Journal
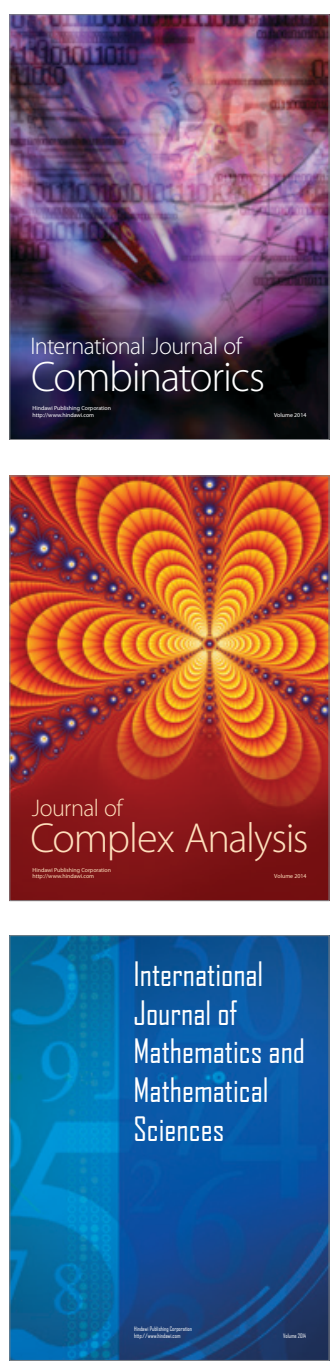
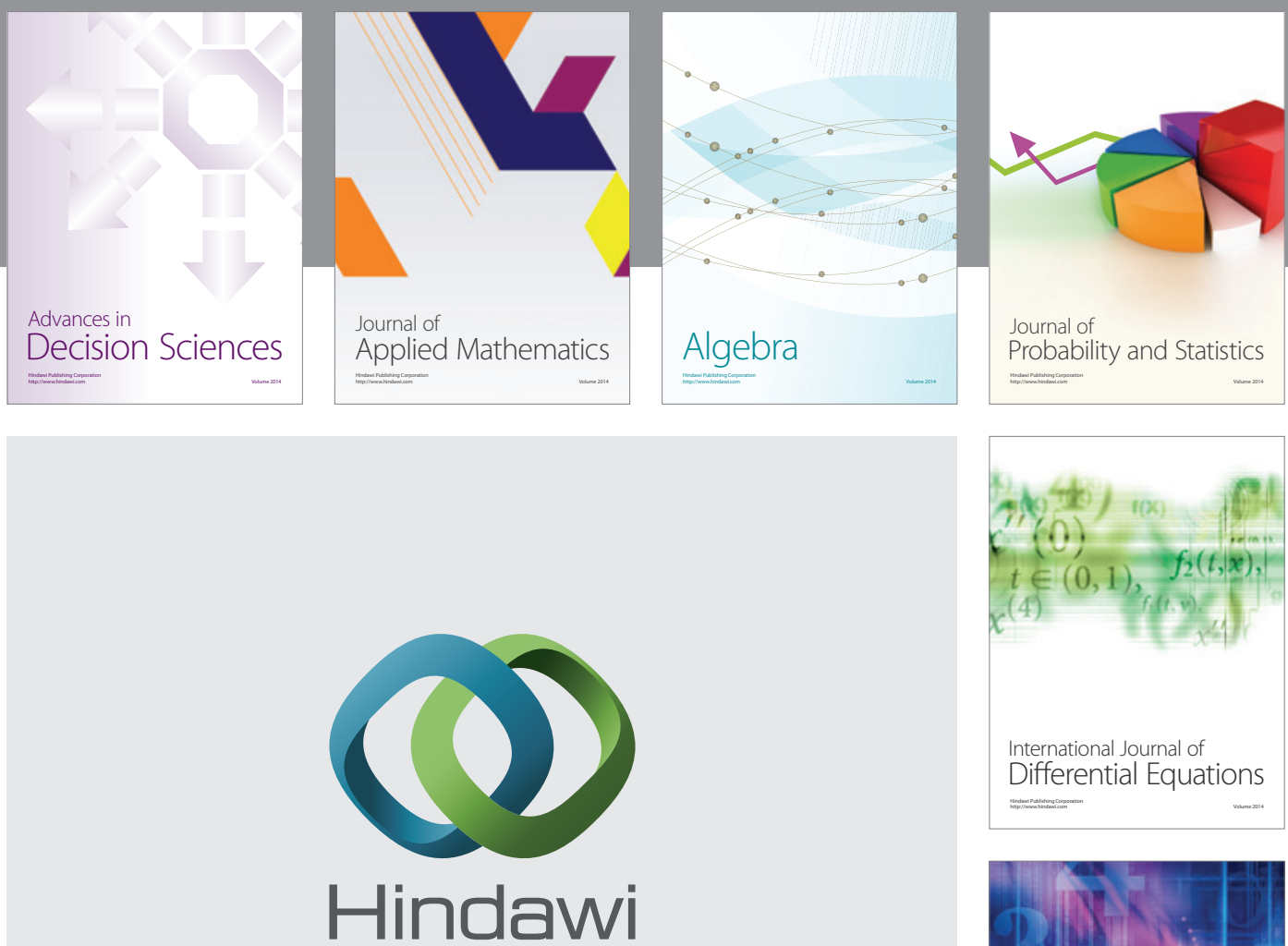

Submit your manuscripts at http://www.hindawi.com
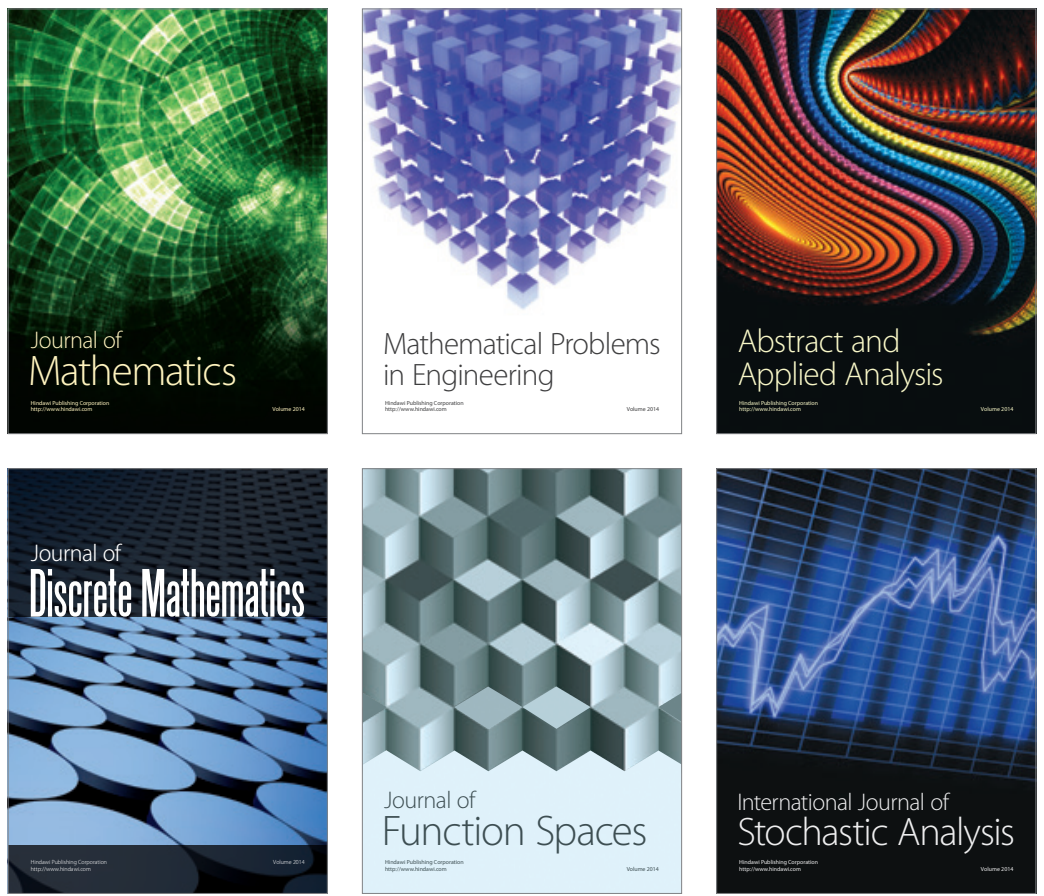

Journal of

Function Spaces

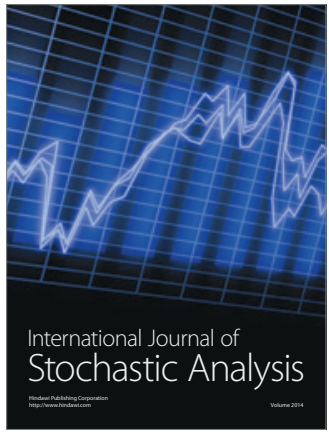

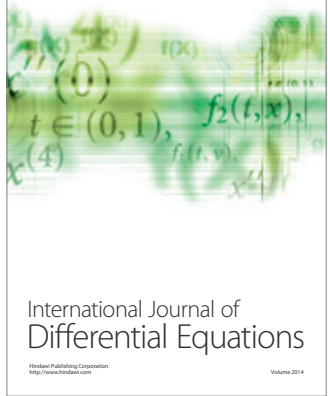
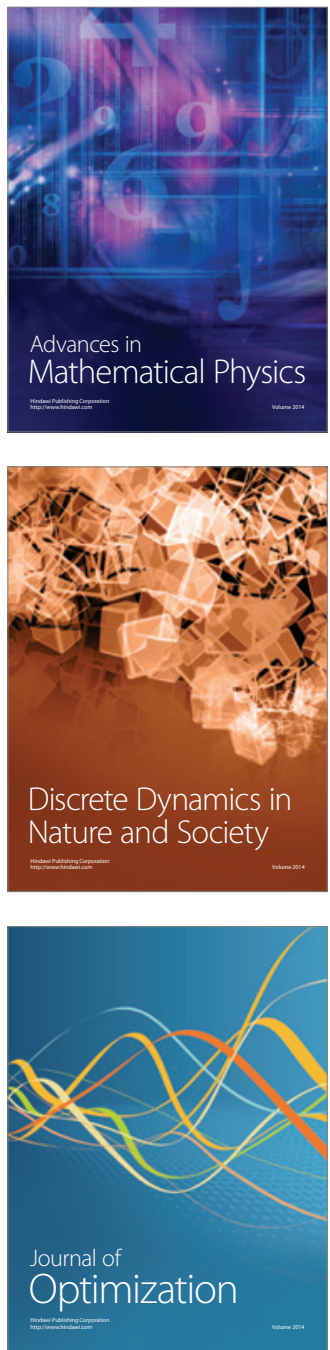